\section{Fieber im Gepäck}

- Mit einer Chance von 45/100.000 Urlaubern kommt das Denguefieber mit von der Thailand-Reise: Im Januar 2013 wurde mit 36 Infektionen ein neuer Höhepunkt erreicht.

- Ca. 64\% der 562 Malaria-Fälle die 2011 gemeldet wurden, waren Reise-Souvenirs; damit setzte sich der 2010 festgestellte Anstieg allerdings nicht fort.

- 2011 war 10-mal Leishmaniose mit im Reise-Rückgepäck: die kutane Leishmaniose kam zweimal mit von Mallorca, fünfmal aus Südamerika und einmal aus Ghana. Zwei viszerale Leishmaniosen kamen je einmal mit aus Georgien/Kasachstan und der Dominikanischen Republik.

RKI, Epidemiologisches Bulletin 2012, 43:429-40

\section{Rotavirus-Impfung für alle Säuglinge}

Die STIKO empfiehlt ab August 2013 die Rotavirus-Impfung für alle Säuglinge vor dem sechsten Lebensmonat. Bereits seit 2006 sind in Deutschland zwei Lebendimpfstoffe gegen Rotaviren (RV) für Säuglinge bis zur vollendeten 24. bzw. 26 . Lebenswoche zugelassen. Bislang wurde die Impfung gegen Rotaviren im Säuglingsalter von der Ständigen Impfkommission (STIKO) allerdings nicht empfohlen. Das wird sich bald ändern: Ab August dieses Jahres sollen laut STIKO Säuglinge vor dem sechsten Lebensmonat nun doch geimpft werden.

21. Jahrestagung der Deutschen Gesellschaft für Pädiatrische Infektiologie (DGPI), 2013, Würzburg

\section{Hurra: „Down Under“ warzenfrei!}

Das australische Impfprogramm gegen humane Papillomviren (HPV) hat Genitalwarzen in einem Ausmaß dezimiert, das selbst die beteiligten Forscher überrascht. Mitte 2007 ist in Australien ein nationales Impfprogramm aufgelegt worden, in dem 12- bis 13-jährige Mädchen kostenlos mit einer tetravalenten HPV-Vakzine geimpft werden können. In den Jahren 2007 bis 2009 gab es zudem ein Nachholprogramm für 13- bis 18-jährige Schülerinnen und für 18 - bis 26-jährige Frauen. Ein Wissenschaftlerteam um Hammad Ali, Sydney, hat nun untersucht, welche Auswirkungen das
Programm auf das Auftreten von Genitalwarzen hat. Verglichen wurden dabei die Zeiträume von 2004 bis 2007 - also vor Einführung der Impfung - und 2007 bis 2011. Die Ergebnisse verblüfften sogar die Forscher. Wurden 2007 noch bei 11,5\% der Frauen unter 21 Jahren Kondylome diagnostiziert, waren es 2011 noch gerade einmal 0,85\% - ein Rückgang um 92,6\%. Ältere Frauen hatten keinen Nutzen von der Impfung, was das Auftreten von Genitalwarzen betraf.

(rb)

Ali Het al, BMJ 2013, doi: 10.1136/bjm.f2032.

\title{
Salmonellengefahr: Hühner überholt
}

Vor Jahren machten Salmonellen aus Eiern und Geflügel Schlagzeilen. Das scheint vorbei zu sein. Der Verzehr von Geflügelfleisch ist nicht mehr das größte Risiko für eine Salmonellose - riskant sind mittlerweile andere Lebensmittel. Vor allem Schweinefleisch ist zunehmend mit Salmonelleninfektionen assoziiert, wie eine Fall-Kontroll-Studie des Niedersächsischen Landesgesundheitsamtes aus Niedersachsen zeigt. Sowohl rohes Schweinemett als auch Schweinemettwurst waren in den univariaten und multivariaten Analysen mit einem erhöhten Salmonel-
lose-Risiko assoziiert. Befragt wurden 1901 Salmonellose-Patienten und zum Vergleich 680 Personen mit einer Rotavirus-Infektion sowie 198 gesunde Kontrollpersonen. Von Schweinemett ging vor allem eine Gefahr für Infektionen mit Salmonella Typhimurium DT 193 aus. Mettwurst war häufiger ein Vehikel für S. Typhimurium DT 104. Auch wer gerne grillt, im Freien isst, auf Auslandsreisen geht oder viele Tierkontakte hat, hatte ein erhöhtes Risiko für Salmonellosen. (dn)

Ziem D et al, Gesundheitswesen 2013, 75 - P18

\section{Von HWI bis HIV}

Harnwegsinfekte sind zum Glück meist harmlos und von kurzer Dauer. Doch was tun, wenn ein Patient Risikofaktoren mitbringt? Muss eine asymptomatische Bakteriurie dann sofort behandelt werden? Und ab wann ist eine Urinkultur notwendig? Antworten auf neun brennende Fragen rund um die Zystitis finden Sie auf Basis der S3-Leitlinie in unserem Schwerpunkt „Infektionen“, in dem sich außer fiesen Blasenkeimen auch HI-Viren, Bettwanzen und andere Plagegeister tummeln. Viel Spaß beim Stöbern!

Claudia Daniels Redakteurin

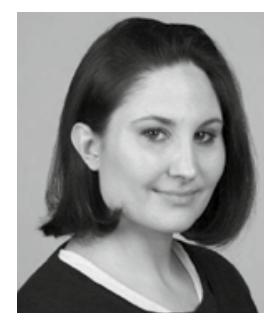

\section{zur Sorge}

Bei der Tuberkulose gibt es in Deutschland keine Entwarnung, betont das Robert Koch-Institut (RKI). Nach den jüngsten Zahlen erkrankten 2011 in der Bundesrepublik 4317 Menschen daran, 162 sind gestorben. Im Vergleich zu 2010 mit 4388 registrierten Erkrankungen und 161 Toten sind die Zahlen damit nahezu unverändert geblieben. Bis 2008 war die Inzidenz viele Jahre deutlich zurückgegangen, teilt das RKI zum Welttuberkulosetag am 24. März mit. Bei 2\% der TB-Kranken werden multiresistente Keime isoliert, bei knapp 12\% sind die Erreger resistent gegen ein Medikament, auch diese Zahlen stagnieren. Gewarnt wird vor allem auch vor extrapulmonaler TB. Tuberkulose trifft bei uns besonders oft Männer ab 40 Jahre. In Städten gibt es deutlich mehr Fälle als auf dem Land. Knapp die Hälfte der Betroffenen sind im Ausland geboren. Bei Kindern und Jugendlichen unter 15 Jahren ist die Erkrankungsrate zwar niedrig, die Zahlen steigen aber an, von 142 (2009) über 160 (2010) auf 179 (2011). Da Kinder sehr anfällig für die Infektion sind, wird nach Kontakt mit einem TBKranken dringend eine Untersuchung empfohlen mit Infektionsdiagnostik und Thoraxröntgen-Untersuchung.

(wg)

Ärzte Zeitung, 24.03.2013 